\title{
Measurements of GaN-Based Heterostructures with Electron Beam Induced Current
}

\author{
K.L. Bunker, J.C. Gonzalez, A.D. Batchelor, P.E. Russell \\ Materials Science and Engineering Department and Analytical Instrumentation Facility, \\ North Carolina State University, Box 7531, Raleigh, NC 27695
}

These studies involved the application of Scanning Electron Microscopy (SEM) and Scanning Transmission Electron Microscopy (STEM) for electrical characterization of GaN-based heterostructures using Electron Beam Induced Current (EBIC). EBIC measurements provide information on the minority carrier diffusion length and surface recombination velocity of bulk semiconductor layers and devices. In this work, we perform SEM-EBIC measurements on bulk GaN-based heterostructure samples in order to extract minority carrier diffusion lengths and to study the influence of beam voltage on the EBIC results. However, the spatial resolution of SEM based EBIC is fundamentally limited by the size of the electron-hole pair generation volume. Therefore, STEM-EBIC was implemented in order to improve the resolution. The combination of a $200 \mathrm{kV}$ beam and a thin sample $(\sim 100 \mathrm{~nm})$ allows for a reduction in the size of the electron-hole pair generation volume and an improvement in the spatial resolution of the EBIC measurements to the nanometer level.

All EBIC measurements in the SEM and STEM were performed in the cross-sectional configuration, with the electron beam parallel to the plane of the $p$ - $n$ junction of the device. The excess minority carriers created by the electron beam at each point are separated by the built-in electric field of the $\mathrm{p}$ $\mathrm{n}$ junction and swept into an external circuit. Plotting the collected EBIC as function of the electron beam position, an EBIC image can be built. From the peak and tails of the EBIC profile, the $p-n$ junction of the device and the minority carrier diffusion length and surface recombination velocity can be determined, respectively.

Previously, a Hitachi S-3200N SEM was used to obtain the minority carrier diffusion length in a GaN-based heterostructures [1]. A theoretical model that includes the distribution, transport, and collection of the minority carriers created by the electron beam was used to extract the minority carrier diffusion length from a mean EBIC linescan. In the present work, a JEOL 6400 SEM was used to determine the minority carrier diffusion length as well as study the effects of beam voltage on low injection conditions and the resolution of the technique. EBIC images were captured at several different accelerating voltages, ranging from $2-10 \mathrm{kV}$, and mean EBIC profiles were obtained from each image by averaging 250 lines (Figure 1). Theoretical calculations of the carrier concentration showed that low injection conditions were maintained at accelerating voltages above $2 \mathrm{kV}$. However, the determination of the minority carrier diffusion length was limited by spatial resolution at voltages above $6 \mathrm{kV}$ and by surface recombination at voltages below $5 \mathrm{kV}$.

An InGaN quantum well heterostructure was studied in a HD-2000 STEM due to the improvement in the spatial resolution achievable in a STEM. The sample was mounted on a TO header with front and back contacts. The sample was embedded in wax and mechanically polished into a wedge. The wedge was placed on a TEM grid, with the grid making contact to the backside. Focused Ion Beam (FIB) milling was used to create an electron transparent membrane of $\sim 100 \mathrm{~nm}$. A custom STEMEBIC sample holder was designed with two isolated low signal electrical feedthroughs (Figure 2). The relative position of the p-n junction with respect to the thin InGaN quantum well was determined with nanometer precision by comparing averaged EBIC and Z-contrast linescans obtained from the corresponding images (Figure 3). The electrical junction was found to be located $(19 \pm 3) \mathrm{nm}$ from the center of the InGaN quantum well in this particular sample. In addition, EDS analysis allowed for compositional identification of the nanometer layers of the device (Figure 3 ). 
[1] J.C. Gonzalez et al., Applied Physics Letters, 79 (2001) 1567.
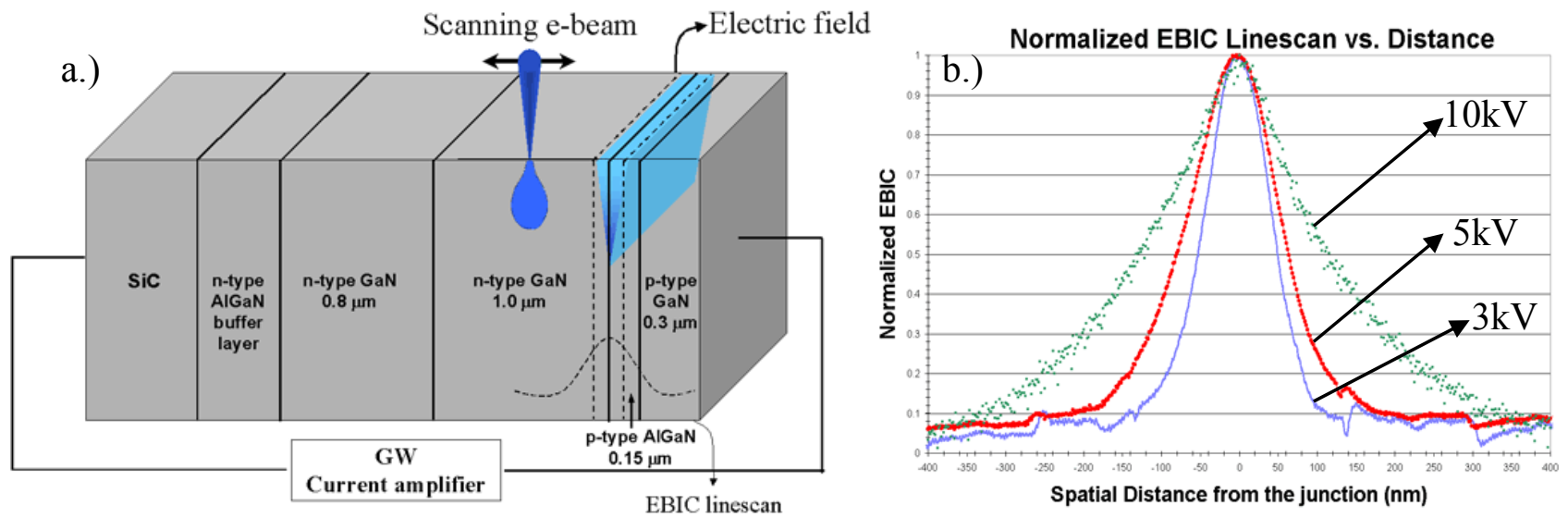

Fig.1 a.) Schematic of the cross-section of the GaN-based LED structure and experimental setup used in the SEMEBIC measurements. b.) Normalized averaged EBIC linescans vs. position for accelerating voltages of 3, 5, and $10 \mathrm{kV}$ obtained in the JEOL 6400 SEM.
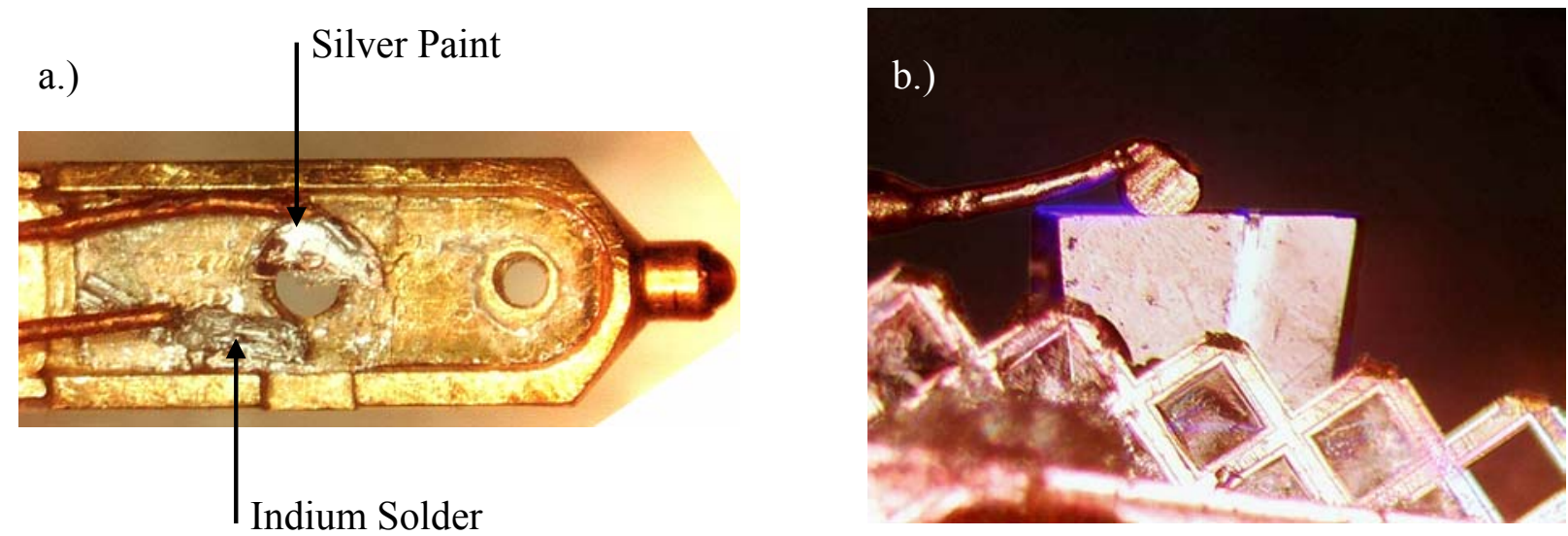

Fig.2 a.) STEM-EBIC customized holder showing the device connected to two electrical feedthroughs with silver paint and Indium solder. b.) GaN-based heterostructure mounted in STEM-EBIC holder and emitting under bias.

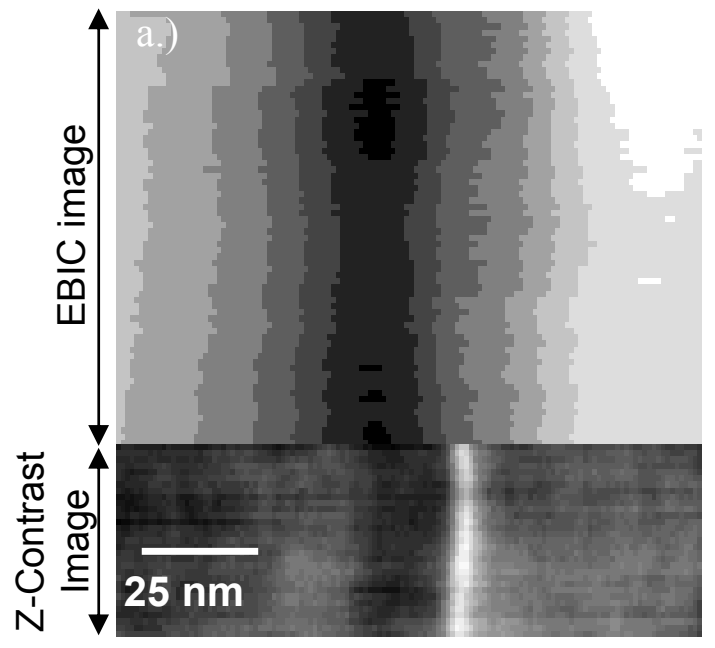

b.)

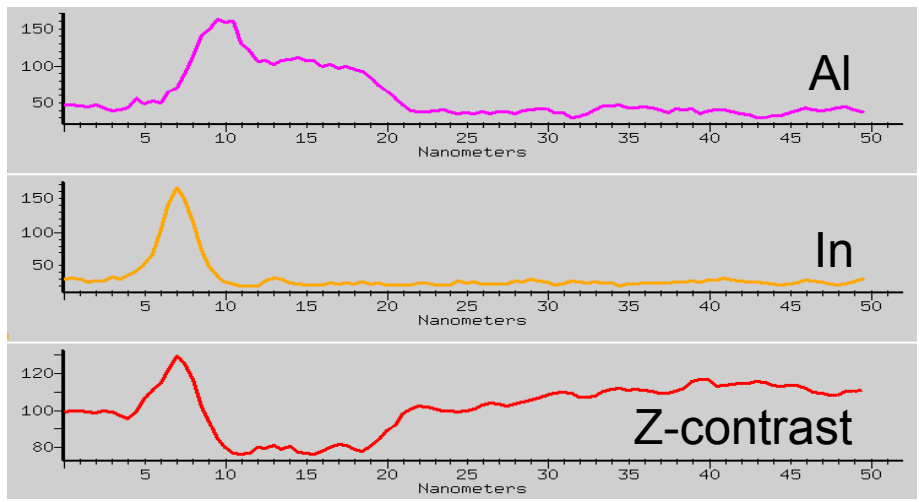

Fig.3 a.) STEM-EBIC and Z-contrast images collected with the Oxford ISIS system. b.) Al, In, and Z-contrast linescans acquired with the Noran Vantage EDS system. 